\title{
Reactionless Control for two Manipulators Mounted on a Cable-Suspended Platform
}

\author{
Roberto Lampariello*, Johann Heindl*, Ralf Koeppe**, Gerd Hirzinger* \\ *Institute of Robotics and Mechatronics \\ ** KUKA Roboter GmbH \\ German Aerospace Center (DLR) \\ 82234 Weßling, Germany \\ Roberto.Lampariello@dlr.de \\ 86165 Augsburg, Germany \\ RalfKoeppe@kuka-roboter.de
}

\begin{abstract}
The dynamics and control of a cable-suspended, two-arm robotic system are developed for an entertainment application. One manipulator arm is controlled to fulfill a user defined task. The second arm is then controlled to compensate for the disturbances on the cable-suspended platform arising from the motion of the first.

Model-based feedforward control, stemming from the momentum conservation equations of a free-floating robot, is developed for the motion compensation problem. Furthermore, due to model uncertainty, sensor-based feedback control is introduced, to account for undesired oscillatory motions of the system. The latter control problem reduces to the dissipation of the oscillatory energy of the system, by means of adequate robot control. Both control methods are implemented and tested on an experimental set-up.
\end{abstract}

\section{INTRODUCTION}

Cable-suspended robots have a wide application for manipulation in large workspaces and where actuation needs to be performed on targets difficult to reach from ground. Examples include inspection and repair in shipyards or transport of externally suspended loads on helicopters [1], [2]. The problems of path tracking and of oscillation damping are then of interest. Control may be performed with direct actuation of the suspension cables [1], [2], or as proposed here, with a momentum compensating element, such as a suitably actuated mass. A particular case is that of using a robot manipulator. This adds interesting aspects, related to entertainment robotics and to multiple-arm free-floating robot control.

This work develops a control method for a cablesuspended, two-arm robotic system, thought for an entertainment application. Two six-degree-of-freedom manipulators are mounted on the opposite faces of a suspended platform, parallel to the ground (see Fig. 1). The goal of the control is to allow one robot, the master, to perform a user-defined six-degree-of-freedom tracking task, such as dusting a car positioned on an elevated platform beneath it. The second robot, the slave, then compensates for any disturbances on the platform which arise from the motion of the master. The desired end result is that the platform remains stationary during robot operation.

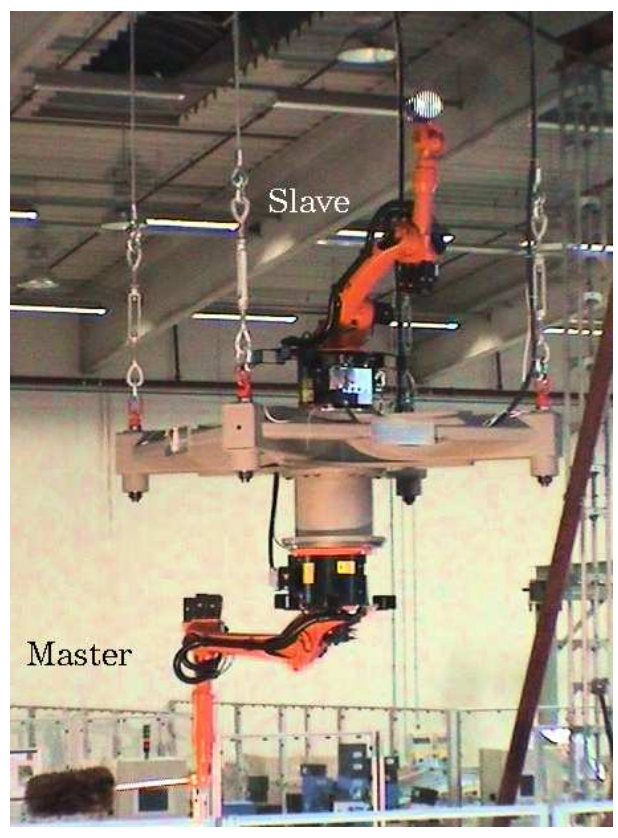

Fig. 1. Experimental set-up of two-arm cable-suspended system: the master is provided with a long duster, the slave with a heavy dumbbell

This control problem has two main components: the first relates to the ideal motion compensation of the two robots, which can be solved with a model-based approach and freefloating robot dynamics; the second relates to the suppression of the system oscillations, which here may arise from model uncertainty or implementation errors, solved with feedback control.

The developed control method is applied to the experimental set-up shown in Fig. 1. The two 6 d.o.f. robot arms have a distinctive upper/lower elbow arm structure, giving rise to a compensation problem also recognized in [3] to be difficult. The added complexity arising from the oscillatory motion element, is treated with a control law based on passivity.

The paper is divided into four subsections: the first describes some related bibliography, the second the theoretical aspects of the control methods, the third the implementation aspects with a description of the experimental set-up and the 
fourth the experimental results. The paper is closed with a conclusions section.

\section{BIBLIOGRAPHY}

Some works of relevance to the compensation control problem include those on free-flying robot dynamics, as in [4] and [5]. In the first, a spatial dynamic model for a multiplearm free-flying robot with reaction wheels is derived. Motion control of the 6 d.o.f. arms is then addressed, operating one to trace a given path and a second to minimize the operational control torque of the reaction wheels, to maintain the attitude of the base body fixed. The formulation of the solution is based on the inverse kinematics on acceleration level. In [5], the full planar compensation problem is considered for a robot with two three-joint arms also extending in the plane. The momentum conservation equations are used and emphasis is given to avoiding singularities of the compensating robot. However, in all cases the joint kinematic and dynamic constraints, which strongly limit the workspace of the compensating robot, are not addressed.

In [6] the inverse kinematics problem for a single-arm freeflying system is analyzed in detail, based on a momentum conservation formulation, although again only attitude motion of the spacecraft is of interest. On the vibration suppression by means of a robot, in [3] the problem of end-effector path tracking and of vibration suppression for macro-mini manipulators is addressed. In both cases, solutions are provided with use of the robot reaction null space, which is not applicable here since the tasks of the master robot are generally sixdimensional.

\section{METHOD}

The equations of motion of a two-arm free-floating robot are first described. For the purpose of the motion compensation control problem, these are then related to the momentum conservation equations for the equivalent system. The equations of the cable suspended system are then formally described. Finally, based on these derivations, the theory for both aforementioned control problems is presented.

\section{A. Equations of motion}

1) Dynamic equations of the free-floating system: Consider the system depicted in Fig. 2. The inertial frame of reference is denoted by $\left\{O^{I}, \underline{\mathbf{e}}^{I}\right\}$. Its relation to the platform body frame of reference $\left\{O^{0}, \underline{\mathbf{e}}^{0}\right\}$ is described by the position vector $\underline{\mathbf{r}}^{0}$ and the orientation matrix $\mathbf{A}^{0}$, parameterized for example by Euler angles $\phi^{0}=\left[\phi_{1}^{0}, \phi_{2}^{0}, \phi_{3}^{0}\right]$, and such that $\underline{\mathbf{e}}^{0}=\mathbf{A}^{0} \underline{\mathbf{e}}^{I}$. Vector quantities are here expressed in the inertial coordinate frame, such that for example $\underline{\mathbf{r}}^{0}=\mathbf{r}^{0} \underline{\mathbf{e}}^{I}$.

The configuration of the $i^{\text {th }}$ robot is described by the column matrix $\boldsymbol{\theta}_{i}$, the elements of which describe the $j^{\text {th }}$ revolute joint position $\theta_{i}^{j}$, measured relative to an arbitrary initial reference configuration.

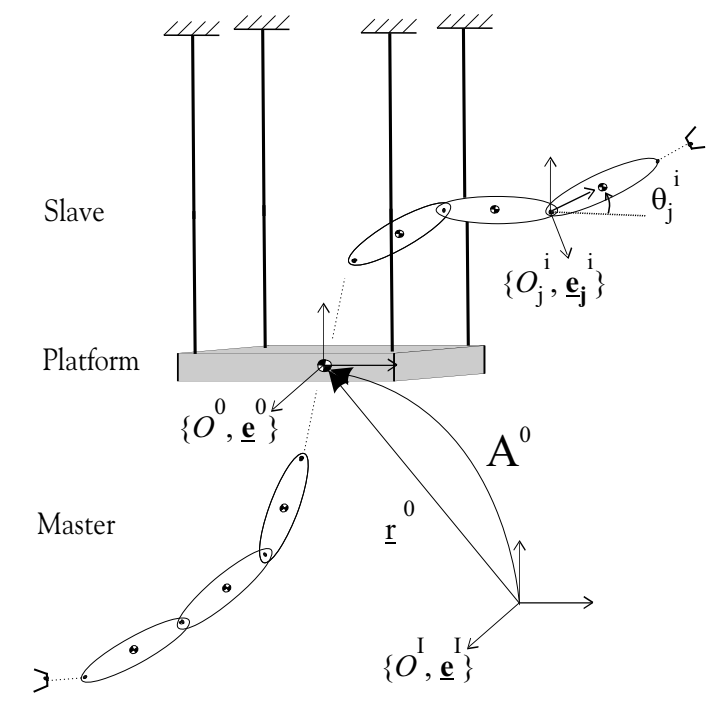

Fig. 2. Configuration and reference frames of the cable-suspended robot

Considering now the free-floating robot dynamics, i.e. omitting gravity and the suspension cables, let the platform state variables be collected as follows:

$$
\mathbf{y}_{I}=\left[\mathbf{r}^{0}, \phi^{0}\right]^{T} \quad \mathbf{y}_{I I}=\left[\mathbf{v}^{0}, \boldsymbol{\omega}^{0}\right]^{T},
$$

where $\mathbf{y}_{I}$ and $\mathbf{y}_{I I}$ are the position and velocity state variables respectively. The formal equation structure can then be written as follows [4]:

$$
\left[\begin{array}{c}
\mathbf{M}_{p} \mathbf{M}_{p r_{1}} \mathbf{M}_{p r_{2}} \\
\mathbf{M}_{p r_{1}}^{T} \mathbf{M}_{r_{1}} \mathbf{M}_{r_{1} r_{2}} \\
\mathbf{M}_{p r_{2}}^{T} \mathbf{M}_{r_{1} r_{2}}^{T} \mathbf{M}_{r_{2}}
\end{array}\right]\left[\begin{array}{c}
\dot{\mathbf{y}}_{I I} \\
\ddot{\boldsymbol{\theta}}_{1} \\
\ddot{\boldsymbol{\theta}}_{2}
\end{array}\right]+\left[\begin{array}{c}
\mathbf{C}_{p} \\
\mathbf{C}_{r_{1}} \\
\mathbf{C}_{r_{2}}
\end{array}\right]=\left[\begin{array}{c}
\mathbf{0} \\
\boldsymbol{\tau}_{1} \\
\boldsymbol{\tau}_{2}
\end{array}\right]
$$

or

$$
\mathbf{M}\left[\begin{array}{c}
\dot{\mathbf{y}}_{I I} \\
\ddot{\theta}_{1} \\
\ddot{\boldsymbol{\theta}}_{2}
\end{array}\right]+\mathbf{C}=\tau,
$$

where $\mathbf{M}$ is the configuration dependent inertia matrix, $\mathbf{C}$ the configuration dependent vector of centrifugal and Coriolis terms and $\tau$ the vector of external actions acting on the degrees of freedom $\mathbf{y}_{I}, \boldsymbol{\theta}_{1}, \boldsymbol{\theta}_{2}$ of the whole system. Furthermore, the subscripts $p, r_{1}$ and $r_{2}$ relate to the platform, the master robot and the slave robot respectively, such that, for example, $\mathbf{M}_{p} \in R^{(3 \times 3)}$ is the inertia matrix which relates to the platform.

It is convenient here to bring out the dynamic coupling between the robots and the platform. As such, from Eq. (2) it follows that:

$$
\mathbf{M}_{p} \dot{\mathbf{y}}_{I I}+\mathbf{C}_{p}=-\mathbf{M}_{p r_{1}} \ddot{\boldsymbol{\theta}}_{1}-\mathbf{M}_{p r_{2}} \ddot{\boldsymbol{\theta}}_{2} .
$$

2) Linear and angular momentum equations: Consider now again the case where gravity and the cables are absent. This is the free-floating case seen above, for which the laws 
of conservation of linear and angular momentum yield the following relationship between the platform and the robot velocities [4]:

$$
\mathbf{M}_{p}\left[\begin{array}{c}
\mathbf{v}^{0} \\
\boldsymbol{\omega}^{0}
\end{array}\right]=-\mathbf{M}_{p r_{1}} \dot{\boldsymbol{\theta}}_{1}-\mathbf{M}_{p r_{2}} \dot{\boldsymbol{\theta}}_{2}
$$

assuming zero initial momentum. This equation, which is first integral of Eq. (3), can be rewritten as:

$$
\left[\begin{array}{c}
\mathbf{v}^{0} \\
\boldsymbol{\omega}^{0}
\end{array}\right]=\mathbf{J}_{1} \dot{\boldsymbol{\theta}}_{1}+\mathbf{J}_{2} \dot{\boldsymbol{\theta}}_{2}
$$

where $\mathbf{J}_{i}$ is a Jacobian matrix of the $i^{t h}$ manipulator, relating base body and joint motion.

3) Dynamic equations of the cable-suspended system: Finally, introducing now the cables and gravity, the final equations of the system formally become:

$$
\mathbf{M}_{p} \dot{\mathbf{y}}_{I I}+\mathbf{C}_{p}=-\mathbf{M}_{p r_{1}} \ddot{\boldsymbol{\theta}}_{1}-\mathbf{M}_{p r_{2}} \ddot{\boldsymbol{\theta}}_{2}+\mathbf{G}+\mathbf{F}_{c},
$$

where the time dependent matrix $\mathbf{G}$ represents the gravity force vector and $\mathbf{F}_{c}$ the vector of cable forces (always positive) respectively.

For the resolution of these equations, useful for simulation purposes, the cables can be modeled as elastic springs. However, the dynamics of the cables elasticity is not of interest here, as it is assumed that their elongation is negligible. Suitable choice of the stiffness parameters in the spring model will give the desired physical behavior, although the resulting numerical stiffness of the equations leads to long computational times.

Note that the above formulations are also useful for the fact that the robots are controlled through their built-in industrial controllers. As such, the control commands are designed at a joint velocity level, rather than at a torque level. This gives rise to delays in the controller performance, as described in the results section.

\section{B. Feedforward Compensation Control}

For the compensation control problem, the system may be idealized as free-floating. The assumption is in fact made that it is initially at rest. In case of perfect knowledge of the system parameters, a reactionless motion of the two robots can be determined with a model-based approach. In fact, if the slave robot can perfectly compensate for the actions of the master on the platform, then the latter will ideally always remain stationary. Any diversion from this is therefore seen as an error, to be compensated with the feedback control action described in section III-C.

1) Free-floating system: Following is the description of the compensation motion control for the general unconstrained case. Consider the conservation of the linear and angular momentum of a free-floating system, expressed in Eq. (4). The solution to the six-dimensional motion compensation problem, where $\dot{\boldsymbol{\theta}}_{1}=\dot{\boldsymbol{\theta}}_{1}(t)$ is an assigned function in time, is given by the condition:

$$
\left[\begin{array}{l}
\mathbf{v}^{0} \\
\boldsymbol{\omega}^{0}
\end{array}\right]=\mathbf{0}
$$

and Eq. (4) simply yields:

$$
\dot{\boldsymbol{\theta}}_{2 s o l}=-\mathbf{M}_{p r_{2}}{ }^{-1} \mathbf{M}_{p r_{1}} \dot{\boldsymbol{\theta}}_{1} .
$$

Such a solution always exists as long as matrix $\mathbf{M}_{p r_{2}}$ is non singular. As we will see, however, the physical constraints on the system (joint actuation limits) are such that only a very limited set of these solutions are practically feasible.

2) Constrained free-floating system: For the constrained cable-suspended system, the formulation is modified as follows. Since, by applying suitable operational constraints on the robots, the platform is constrained in the vertical translational and in the horizontal in-plane rotational motions, either by the cables, or by the weight of the entire system, then only the remaining degrees of freedom need to be compensated for. In fact, for the vertical translational motion, the robot inertial forces on the platform would have to overcome the weight of the system (see Eq. (6)). Likewise, for the inplane rotational motions, the robot inertial moments about a given pivoting side of the platform would have to exceed that generated by the weight of the system. Due to the large mass of the experimental system used, these limitations turn out to be very unrestrictive and were therefore ignored.

To solve the constrained compensation control problem, Eq. (4) can then for example be written as follows:

$[3 \times 3] \mathbf{M}^{\prime}\left[\begin{array}{c}v_{x}^{0} \\ v_{y}^{0} \\ \omega_{z}^{0}\end{array}\right]=-{ }_{[3 \times 6]} \mathbf{M}_{p r_{1}}{ }^{\prime} \dot{\boldsymbol{\theta}}_{1}-_{[3 \times 6]} \mathbf{M}_{p r_{2}}{ }^{\prime} \dot{\boldsymbol{\theta}}_{2}$,

where the primed matrices have been obtained by the relative terms in their fully six-dimensional counterparts (their dimensions are defined by the lower left-subscript). In this example, all joints of the slave robot are used (more will be said about this point in subsection III-B.4). Therefore, setting the left-hand side to zero, as required, gives rise to a least squares problem.

3) Full quadratic programming formulation: In practice, kinematic and dynamic supplementary constraints on the slave robot motion enter into the problem. These are inequality constraints, which relate to the joint positions, velocities and accelerations. A quadratic programming problem can then be formulated, where the kinetic energy of the slave robot $\Gamma$ is to be minimized:

$$
\min _{\dot{\boldsymbol{\theta}}_{2}} \Gamma\left(\dot{\boldsymbol{\theta}}_{2}\right)=\frac{1}{2} \dot{\boldsymbol{\theta}}_{2}^{T} \mathbf{M}_{r_{2}} \dot{\boldsymbol{\theta}}_{2},
$$

where $\mathbf{M}_{r_{2}}$ is the slave robot inertia matrix, and with equality and inequality constraints as follows:

$$
\mathbf{M}_{p r_{1}}{ }^{\prime} \dot{\boldsymbol{\theta}}_{1}+\mathbf{M}_{p r_{2}}{ }^{\prime} \dot{\boldsymbol{\theta}}_{2}=\mathbf{0}
$$




$$
\begin{aligned}
& \theta_{2 \min }^{i} \leq \theta_{2}^{i} \leq \theta_{2 \max }^{i} \\
& \dot{\theta}_{2 \min } \leq \dot{\theta}_{2}^{i} \leq \dot{\theta}_{2 \max }^{i} \\
& \ddot{\theta}_{2 \min }{ }_{2} \leq \ddot{\theta}_{2}{ }_{2} \leq \ddot{\theta}_{2 \max }^{i} .
\end{aligned}
$$

The joint positions and accelerations can be linearly related to the velocities by simple numerical integration or differentiation, i.e.

$$
\begin{aligned}
& \theta^{i}{ }_{2}(t)=\theta_{2}{ }_{2}(t-\Delta t)+\dot{\theta}^{i}{ }_{2}(t) \Delta t \\
& \ddot{\theta}^{i}{ }_{2}(t)=\left(\dot{\theta}^{i}{ }_{2}(t)-\dot{\theta}^{i}{ }_{2}(t-\Delta t)\right) / \Delta t,
\end{aligned}
$$

where $\Delta t$ is equal to the sampling rate of $12 \mathrm{~ms}$. As such, for the given optimization problem, the optimal solution $\dot{\boldsymbol{\theta}}_{2}^{*}$ is unique, since the matrix $\mathbf{M}_{r_{2}}$ is positive definite [8]. This solution locally minimizes the weighted norm of the joint velocities, implying that no internal motions result.

4) Compensation limit and kinematic decoupling of the slave robot: It turns out that the physical bounds on the slave robot joints make the range of master motions which can be compensated rather limited. This is due to the robot kinematic configuration, which gives rise to a strong coupling in the joint motions for the actions exerted at the base. Practically, compensating forces or torques alone is simple, but compensating both simultaneously is not. Nenchev also points this out in [3].

This fact can be simply viewed as follows. Consider first the case in which only the first three joints of the slave robot are used, for which now $\mathbf{M}_{p r_{2}}{ }^{\prime} \in R^{(3 \times 3)}$ in Eq. (11), which for our purpose would simply need to be inverted. Furthermore, if we imagine to have a sliding disk in place of the slave robot, actuated in the two translational degrees of freedom of interest, and which is also allowed to rotate about its axis of symmetry, then the same matrix would be diagonal and the compensation limit would be decoupled for each direction. This could actually be an interesting hardware implementation for different industrial applications of this robotic system. Instead, for the robot kinematics shown in Fig. 3, where the first joint is revolute, matrix $\mathbf{M}_{p r_{2}}$ includes off-diagonal elements, which give rise to the strong joint coupling.

A judicious initial slave robot configuration is a first measure to improve the solution of the inverse kinematics problem. This can be chosen by the condition:

$$
\min _{\theta_{2}} \kappa=\frac{\sigma_{1}}{\sigma_{2}}
$$

where $\kappa$ is the condition number of matrix $\mathbf{M}_{p r_{2}}$, while $\sigma_{1}$ and $\sigma_{2}$ its largest and smallest singular values respectively. This favors a well-conditioned inverse, for the resolution of Eq. (11).

A further development involves the decoupling of the slave robot kinematics. This in turn involves choosing a particular configuration of the wrist joints. In fact, by selecting joint 5

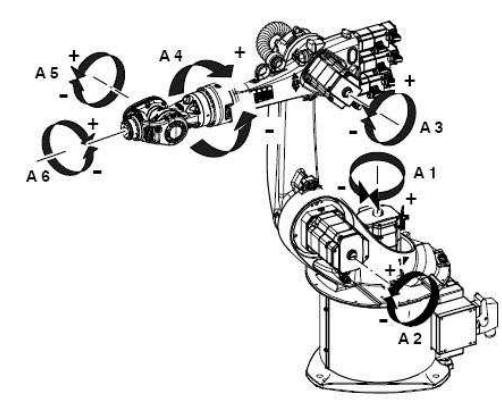

Fig. 3. Kinematics of KUKA robot

such that joint 6 is always aligned with the vertical, i.e. (see also Fig. 3)

$$
\dot{\theta}_{2}^{5}=-\dot{\theta}_{2}^{2}-\dot{\theta}_{2}^{3} \quad \dot{\theta}_{2}^{4}=0,
$$

ensures that the latter's action only applies to the rotational degree of freedom of the platform. This way, joints 1,2 and 3 can be applied to compensate for the translation disturbances on the system and the decoupled joint 6 can then deal with the remaining rotational element.

Mathematically the solution is as follows. Let Eq. (11) now become

$$
{ }_{[3 \times 6]} \mathbf{M}_{p r_{1}}{ }^{\prime} \dot{\boldsymbol{\theta}}_{1}+{ }_{[3 \times 4]} \mathbf{M}_{p r_{2}}{ }^{\prime \prime}{ }_{[1 \times 4]} \dot{\boldsymbol{\theta}}_{2}{ }^{\prime}=\mathbf{0},
$$

where the double-primed matrix $\mathbf{M}_{p r_{2}}$ " has dimensions $(3 \times 4)$ and $\dot{\boldsymbol{\theta}}^{\prime}{ }_{2}$ is a $(4 \times 1)$ vector, containing the first three and the last robot joint velocities (the effect of joint 5 is here neglected for simplicity). The conditions in Eq. (18) guarantee that the first two rows of Eq. (19) can be solved independently of $\dot{\theta}_{2}^{6}$, giving a solution for joint velocities $\dot{\theta}_{2}^{1}, \dot{\theta}_{2}^{2}$ and $\dot{\theta}_{2}^{3}$. Then, the last row of Eq. (19), which includes all terms of $\dot{\boldsymbol{\theta}}_{2}^{\prime}$, can be solved in $\dot{\theta}_{2}^{6}$ for the previously found solution of the first three joints. As will be shown in the results section, this configuration was found to be successful for a wider range of motions of the master robot.

Equations (10)-(14) (or (19) in place of (11)) can be solved for subsequent time steps to obtain a solution for a given master robot motion. The solution can be used to partly validate the feasibility of the compensation, with respect to the motion constraints. The global optimization of kinematic redundancy was however not addressed here.

\section{Feedback Oscillation-Suppression Control}

The solution in the previous section is only suitable for feedforward control. A feedback control is necessary to account for modeling and implementation errors during the execution of the task which, due to the effect of gravity, give rise to an undesired oscillatory motion of the platform. The feedback error then consists of the platform motion, the measurements of which are provided by a camera system 
placed above it.

Here, the aim of the controller is to add any necessary compensation, via extra slave robot actuation, to account for the platform motion disturbance. We first consider the simpler case where the slave robot only has to dissipate the energy of the system initially set in oscillation. Then, the extension to the dual task of compensating for the master robot as well as the oscillation suppression is addressed.

Let us then analyze the first problem in the framework of passive systems and consider the specific case of passivitybased position control, as described in [7]. The Hamiltonian of the platform is

$$
\mathbf{H}_{p}=\frac{1}{2} \dot{\mathbf{y}}_{I}^{T} \mathbf{M}_{p} \dot{\mathbf{y}}_{I}+\mathbf{U}\left(\mathbf{y}_{I}\right),
$$

where $\mathbf{y}_{I}$ and $\dot{\mathbf{y}}_{I}$ represent the platform position and velocity states respectively (cf. Eq. (1)), $\mathbf{M}_{p}$ its inertia matrix (cf. Eq. (6)) and $\mathbf{U}\left(\mathbf{y}_{I}\right)$ its potential energy (this includes the gravity force term $\mathbf{G}$ and the term of cable forces $\mathbf{F}_{c}$ ).

It is straightforward to state that the platform is in itself a passive system. In fact, Eq. (20) shows that its total energy has a bounded minimum in the stationary position of interest, since the potential energy is referenced to an arbitrary constant. This position, without loss of generality, may be given by $\mathbf{r}^{0}=0, \phi^{0}=0$, for which the gravity force term $\mathbf{G}$ is zero. Furthermore, if the condition holds that $\mathbf{v}^{0}=0, \boldsymbol{\omega}^{0}=0$, then the system is in equilibrium since no actions result.

The Hamiltonian $\mathbf{H}_{p}$ also satisfies the following equation:

$$
\dot{\mathbf{H}}_{p}=\dot{\mathbf{y}}_{I}^{T} \mathbf{v},
$$

where $\mathbf{v}$ represents the external nonconservative forces and torques. This implies that the platform is passive from the input $\mathbf{v}$ to the output $\mathbf{y}_{I I}$. Damping is then introduced to asymptotically stabilize the system:

$$
\mathbf{v}=-\mathbf{K}_{d} \dot{\mathbf{y}}_{I},
$$

where $\mathbf{K}_{d}>0$ is a diagonal matrix. Equation (21) then becomes

$$
\dot{\mathbf{H}}_{p}=-\dot{\mathbf{y}}_{I}^{T} \mathbf{K}_{d} \dot{\mathbf{y}}_{I} .
$$

From Eq. (6), it follows that the control forces on the platform arising from the robot are

$$
\mathbf{v}=-\mathbf{M}_{p r_{2}} \ddot{\boldsymbol{\theta}}_{2},
$$

(cf. Eq. (6)).

Equating Eq. (22) and (24) gives the sought relationship

$$
\dot{\mathbf{y}}_{I}=\mathbf{K}_{d}^{-1} \mathbf{M}_{p r_{2}} \ddot{\boldsymbol{\theta}}_{2} .
$$

Equation (23) then becomes

$$
\dot{\mathbf{H}}_{p}=-\ddot{\boldsymbol{\theta}}_{2}^{T} \mathbf{M}_{p r_{2}}{ }^{T} \mathbf{K}_{d}{ }^{-1} \mathbf{M}_{p r_{2}} \ddot{\boldsymbol{\theta}}_{2} .
$$

If the matrix product $\mathbf{M}_{p r_{2}}{ }^{T} \mathbf{K}_{d}{ }^{-1} \mathbf{M}_{p r_{2}}$ is always positive- definite, then expression (26) is negative semi-definite, and by use of La Salle's invariance theorem, the asymptotic stability of the equilibrium can be proven [7].

To this purpose, it is a known result that a real symmetric matrix, $\mathbf{B}$, is positive definite if there exists a real nonsingular matrix, $\mathbf{M}$, such that

$$
\mathbf{B}=\mathbf{M}^{T} \mathbf{A} \mathbf{M},
$$

as long as $\mathbf{A}$ is also positive definite [9]. This argument can be extended to the matrix $\mathbf{J}_{2}=\mathbf{M}_{p}{ }^{-1} \mathbf{M}_{p r_{2}}$, since the matrix $\mathbf{M}_{p}$ is positive definite. It is then sufficient that the Jacobian matrix $\mathbf{J}_{2}$ is never singular to satisfy the necessary condition above.

By judicious choice of the gain matrix $\mathbf{K}_{d}$, for a given maximum platform oscillation, the slave robot motions are small enough to ensure that a singularity is never met. This is of course at the expense of the platform motion damping.

From the above reasoning it follows that if Eq. (25) holds, then the platform motion is asymptotically stable. The control input is then chosen as:

$$
\dot{\boldsymbol{\theta}}_{2}=\int_{0}^{t} \mathbf{M}_{p r_{2}}{ }^{-1} \mathbf{K}_{d}\left[\begin{array}{c}
\mathbf{v}^{0}{ }^{m e s} \\
\boldsymbol{\omega}^{0}{ }_{m e s}
\end{array}\right] d t,
$$

where subscript 'mes' refers to measured values.

For the combined control problem, the local solution is chosen as the sum of that from Eq. (11) and that of Eq. (28) (the latter may also be modified by an equivalent expression of $\mathbf{M}_{p r_{2}}$ for the kinematically decoupled case seen in subsection III-B.4).

\section{EXPERIMENTAL SET-UP}

Details of the experimental set-up are given in Fig. 4. Two global cameras placed on the ceiling above the system deliver the position and velocity of the platform via pattern

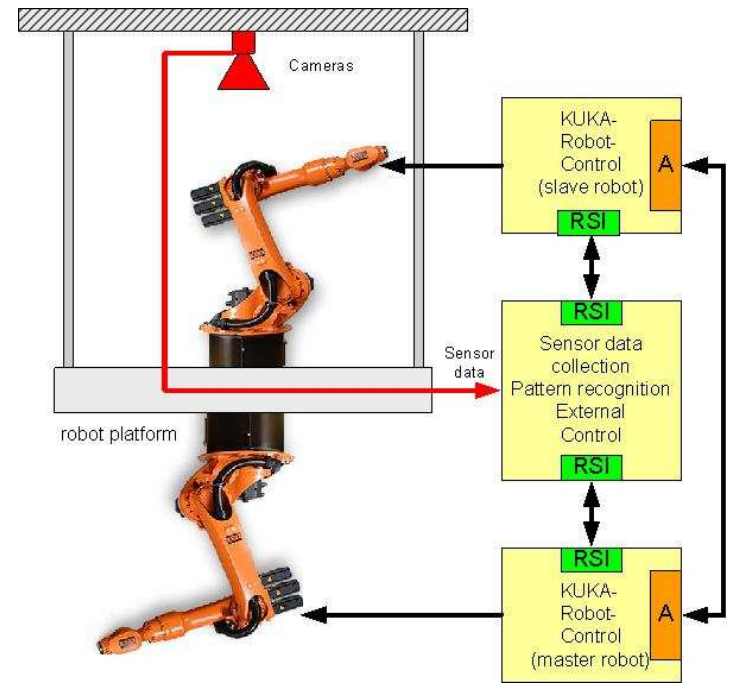

Fig. 4. Details of the experimental set-up 
recognition with a sampling rate of $80 \mathrm{~ms}$. The two KUKA robot controllers (KRC) are configured for a synchronization period of $12 \mathrm{~ms}$, using the KUKA Ethernet-based RoboTeam Technology (shown in Fig. 4 as interface 'A'). The data transfer, also synchronized in a $12 \mathrm{~ms}$ period, between the external sensor computer and the KRC-computers, is realized by the Robot Sensor Interface (RSI).

\section{RESULTS}

A. Reactionless control: comparison between non-decoupled and decoupled robot kinematics
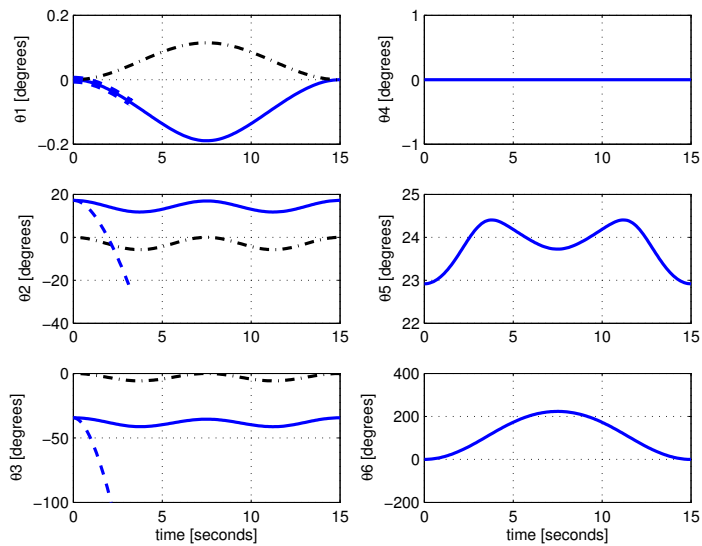

Fig. 5. Comparison between three-joint and decoupled four-joint compensation: dashdot black line - master robot motion; solid blue line - slave robot decoupled motion; dashed blue line - three-joint slave robot (terminated at time $\mathrm{t}=3.4 \mathrm{sec}$. due to joint position constraints)

Fig. 5 shows numerical solutions for the compensation problem with a three d.o.f slave robot, as firstly described in section III-B.4, and for a four d.o.f decoupled-kinematics robot as described by Eq. (19). It is evident that the second solution is more successful and that already for the very small master motion shown, no feasible solution is found in the first case. However, a high velocity of joint 6 is required despite the load with large inertia. In fact, due to the relatively small size of the inertia of the sixth link, a saturation limit on the velocity of the joint of 18 radians/second was introduced.

\section{B. Reactionless control with decoupled robot kinematics}

In Fig. 6 an example master robot maneuver for an entertainment application is shown. The maneuver is for the robot dusting a car placed beneath it. For the first 30 seconds the master is not actuated, to allow for the slave to dissipate any residual oscillatory motion of the system. Note that all master robot joints are actuated within a reasonable range of their workspace.

The effect of the slave robot feedforward compensation is shown in Fig. 7. The poor performance in the rotation can be attributed to the velocity saturation limit of the sixth joint.
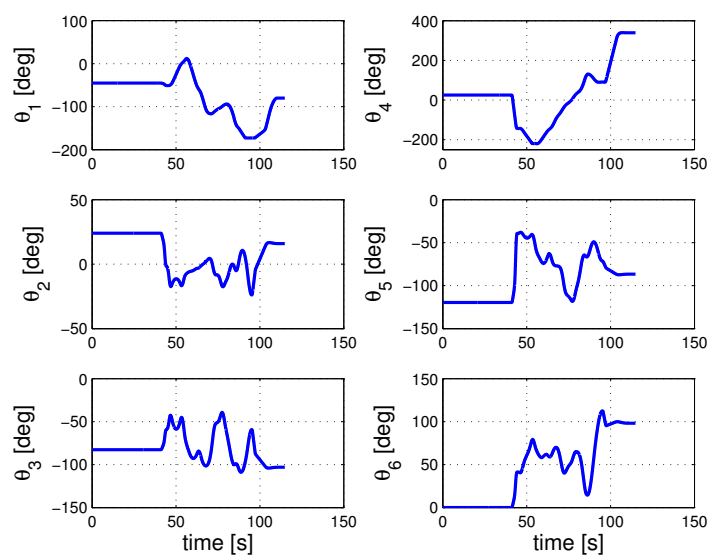

Fig. 6. Master robot joint positions for car-dusting maneuver
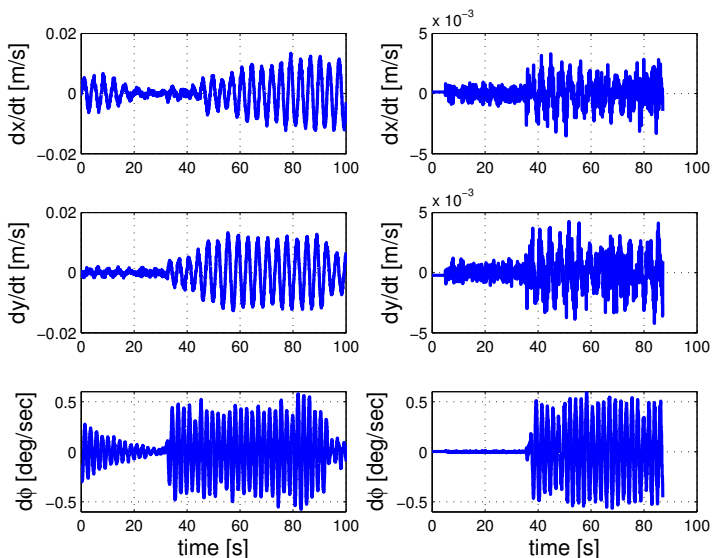

Fig. 7. Platform motion for car-dusting maneuver: left-hand side - no slave robot control; right-hand side - slave robot feedforward control

In Fig. 8 the cases of only feedback and feedforward with feedback are shown. Note that the first case performs better than the second. This is due to the coupling between the platform rotational motion and the translational momentum equations introduced by matrix $\mathbf{M}_{p r_{2}}{ }^{-1}$ in Eq. (28), such that the rotational error is also constrained by the first three joints of the slave robot, instead of only by its last joint.

However, the entertainment element for the observer might also have some say in the choice of one or the other control method. Note that the slave motion is perhaps more interesting in the feedforward-feedback case than in the mere feedback case, as shown in Fig. 9.

Implementation errors may be due to modeling mismatch (parameter uncertainty, some elasticity in the cables), but also in the low-level robot control (note that velocities were commanded) and in the camera time delays. This problem was efficiently overcome by substitution of the platform 

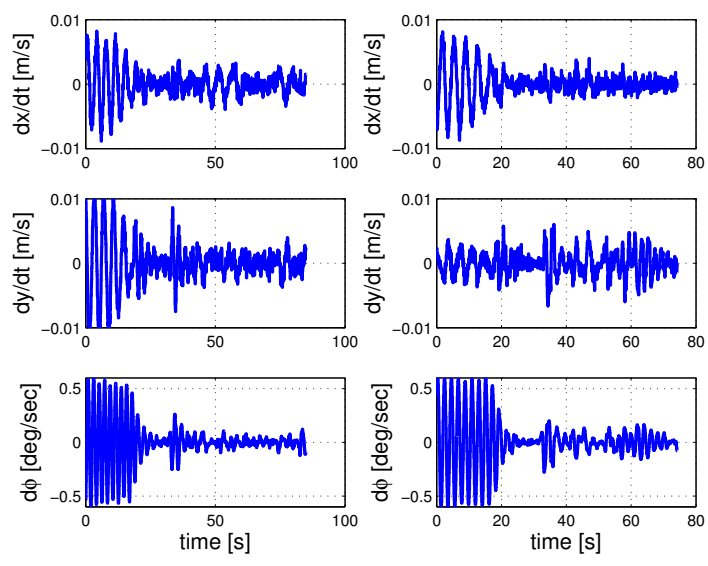

Fig. 8. Platform motion for car-dusting maneuver: left-hand side - slave robot feedback control only; right-hand side - slave robot feedback and feedforward control
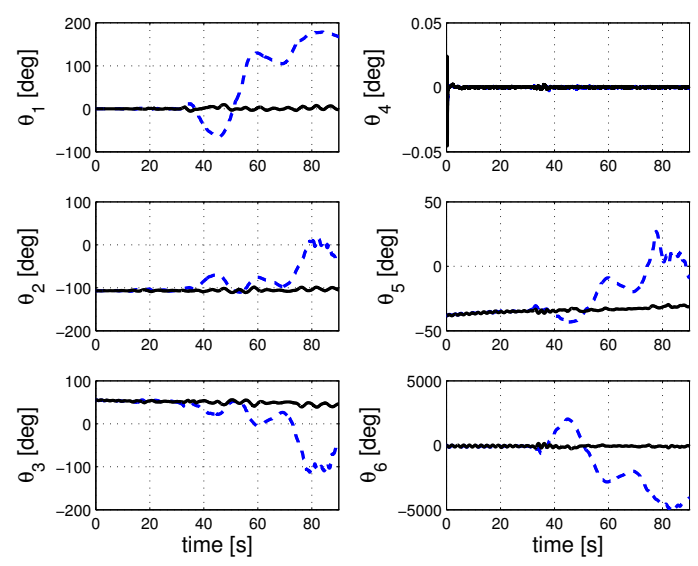

Fig. 9. Slave robot joint positions for entertainment maneuver: dotted blue line - feedforward-feedback control; solid black line - feedback control

position measurements with velocity measurements, thus reintroducing the required phaselag in the control law (c.f. Eq. (28)). This was allowed by the fortuitous ratio between the time delay and the oscillation periods of the system.

\section{Energy dissipation}

Fig. 10 shows examples of the slave robot feedback control for the dissipation of the oscillation energy of the system initially in motion. For the case on the right-hand side, the robot is not actuated, showing the natural dissipation of the system. On the left-hand side, two examples of energy dissipation are given for parameter sets $\mathbf{K}_{d}=[1.0,0,0 ; 0$, $1.0,0 ; 0,0,300.0]$ and $\mathbf{K}_{d}=[0.5,0,0 ; 0,0.5,0 ; 0,0,50.0]$ respectively.
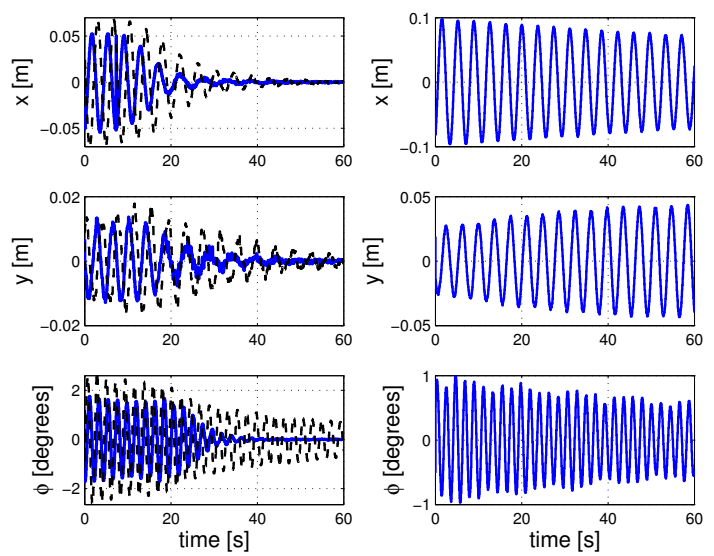

Fig. 10. Energy dissipation control. Right-hand side: no control; Left-hand side: dashed black line - slow dissipation, solid blue line - fast dissipation

\section{CONCLUSION}

A control method was developed for the reactionless control of two robot manipulators, with a distinctive upper/lower elbow arm structure, mounted on a cable-suspended platform. A feedforward component was determined with the momentum conservation equations of a free-floating robot in the plane and with kinematic decoupling of the compensating robot, to reduce the limitations arising from the joint motion constraints. A control law was presented for the dissipation of oscillation energy in the system, based on passivity, to account for the compensation errors arising from model uncertainty and from implementation errors.

\section{REFERENCES}

[1] Alp, A. B., Agrawal, S. K.: Cable Suspended Robots: Feedback Controllers with Positive Inputs, Proceedings of the American Control Conference, 2002.

[2] Oh, S., Ryu, J., Agrawal, S. K.: Dynamics and Control of a Helicopter Carrying a Payload Using a Cable-Suspended Robot, In Proceedings, ASME IDETC/CIE 2005.

[3] Nenchev, D., Yoshida, K., Vichitkulsawat, P., Uchiyama, M.: Reaction Null-Space Control of Flexible Structure Mounted Manipulator System, IEEE Transactions on Robotics and Automation, Vol. 15, No. 6, Dec. 1999.

[4] Yoshida, K., Kurazume, R., Umetani, Y.: Dual Arm Coordination in Space Free-flying Robot, International Conference on Robotics and Automation, Sacramento, California, April 9-11, 1991.

[5] Agrawal, S. K., Shirumalla, S.: Planning Motions of a Dual-Arm FreeFloating Manipulator Keeping the Base Inertially Fixed, Mech. Mach. Theory, Vol. 30, No. 1, pp.59-70, 1995.

[6] Nenchev, D., Umetani, Y., Yoshida, K.: Analysis of a Redundant FreeFlying Spacecraft Manipulator System, IEEE Transactions on Robotics and Automation, Vol. 8, No. 1, Feb. 1992.

[7] Khalil, W., Dombre, E.,Modeling, Identification and Control of Robots, Hermes Penton Science Ltd, 2002.

[8] Fletcher, R.:Practical Methods of Optimisation, John Wiley \& Sons, 1987.

[9] Golub, G. H., Van Loan, C.: Matrix Computations, The Johns Hopkins University Press, p. 141, 1996. 